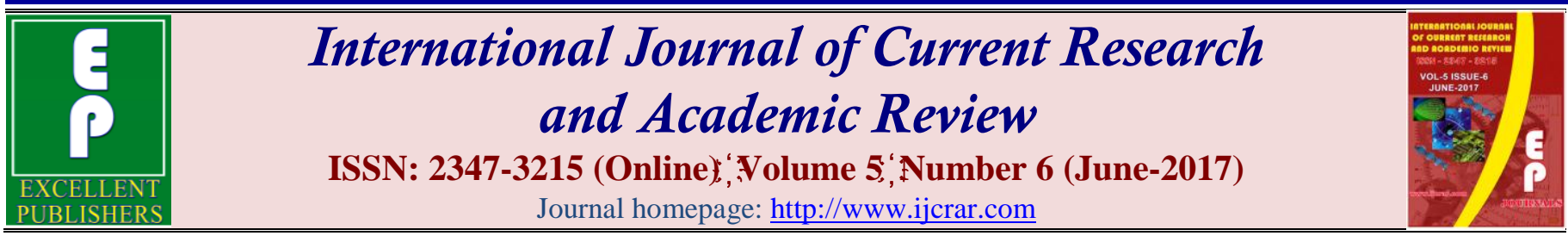

doi: https://doi.org/10.20546/ijcrar.2017.506.004

\title{
Pre-clinical Safety Evaluation of Recombinant Human Granulocyte Macrophage Colony Stimulating Factor (rh GM-CSF)
}

\author{
Bajji Chitra $^{1,2^{*}}$, Durga Bhavani Konga ${ }^{1}$, Pavan Kumar Manikonda ${ }^{1}$, P. Sudhakar ${ }^{2}$, K.R.S. Sambha \\ Siva Rao $^{2}$ and Murali Krishna Reddy Tummuru ${ }^{1}$
}

${ }^{I}$ Virchow Research Center, Hyderabad, INDIA - 500043

${ }^{2}$ Depeartment of Biotechnology, Acharya Nagarjuna University, Nagarjuna Nagar, Guntur-522510, India

*Corresponding author:

\begin{abstract}
Granulocyte-macrophage colony-stimulating factor (GM-CSF), a monomeric glycoprotein produced by macrophages, T cells, mast cells and NK cells naturally. Since, this cytokine is responsible for the survival, proliferation, differentiation and function of myeloid cells, it has been used in vast applications such as cancer and inflammatory diseases. There is huge requirement of this therapeutic drug, recombinant human GM-CSF (rhGM-CSF) has been developed in yeast cells and characterized by various biophysical properties by the present investigators using rDNA technology. The rhGM-CSF has been increased the yield by expressing with T7 promoter and downstream of pelB signal peptide $(2.5 \mathrm{kDa})$ in yeast cells and it can be used as a therapeutic protein where it shows the similar biochemical properties with natural human rhGM-CSF protein (Chitra et al., 2017). Further, to characterize any toxicological symptoms that might be produced in animals and to find recommended dose levels of rhGM-CSF for using as a therapeutic drug, the present investigation has been focused. Acute, sub-acute and chronic toxicity tests are routinely carried out by various pharmaceutical companies in the development of new medicines. Initially, acute study was conducted by administering ten times of the therapeutic dose of rhGMCSF to mice and observed for 15 days to find the pre-terminal morbidity, mortality and any toxic reactions. There are no significant toxicological symptoms or any other adverse reactions observed during the sub chronic study in all males and females, it indicating that rhGM-CSF is safe up to the $325 \mu \mathrm{g} / 20 \mathrm{~g}$ mice and $116.6 \mu \mathrm{g} / \mathrm{kg}$ rabbit dose level. Based on these results, the present investigation suggested rhGM-CSF is safe and further conducting the clinical studies might be helpful for using the rhGM$\mathrm{CSF}$ as a therapeutic drug.
\end{abstract}

\section{Article Info}

Accepted: 05 June 2017

Available Online: 20 June 2017

\section{Keywords}

GM-CSF: Granulocyte-macrophage colony-stimulating factor, Acute toxicity, Sub-chronic toxicity study, Recombinant DNA technology, Clinical chemistry, Hematology.

\section{Introduction}

Granulocyte macrophage colony stimulating factor (GM$\mathrm{CSF}$ ) is a monomeric $23 \mathrm{kD}$ glycoprotein with $4 \infty-$ helical bundle structure produced by macrophages, $\mathrm{T}$ cells, mast cells and NK cells (Metcalf, 1998). It acts as a cytokine and colony stimulating factor 2 (CSF2) that binds to the heterodimeric receptor, which is belonging to type-1 cytokine receptor family (Zhang, 2000). GMCSF activates the inflammatory cascade by stimulating 
the stem cells to generate granulocytes and monocytes which activates macrophages to an increase rapidly in their numbers to facilitate development of the immune system and promotes defense against infections (Paine et al., 2000). This cytokine is responsible for the survival, proliferation, differentiation and function of myeloid cells (Guthridge, 1998). Recently, GM-CSF has been used to regimens for the mobilization of hematopoietic progenitor cells and also essential for maintaining basal hematopoiesis (Fantuzzi, 2003, Cashen et al., 2004). Further, the increase in dendritic cells content and activity following local and systemic GM-CSF administration support a role for GM-CSF as an immune stimulant and vaccine adjuvant in cancer patients (Ferlazzo et al., 2000; Sanoda, 1988).

Recombinant human GM-CSF (rhGM-CSF) has been developed in yeast cells and characterized by various biophysical properties by the present investigators using recombinant DNA technology (Ballinger et al., 2006). The rhGM-CSF has been increased the yield by expressing with $\mathrm{T} 7$ promoter and downstream of pelB signal peptide $(2.5 \mathrm{kDa})$ in yeast cells and it can be used as a therapeutic protein where it shows the similar biochemical properties with natural human rhGM-CSF protein (Chitra et al., 2017).

Further, to characterize any toxicological symptoms that might be produced in animals and to find recommended dose levels of rhGM-CSF for using as a therapeutic drug, the present investigation has been focused. Acute, subacute and chronic toxicity tests are routinely carried out by various pharmaceutical companies in the development of new medicines. Initially, pretesting was conducted by administering rhGM-CSF to mice and observed for 15 days to find the pre-terminal morbidity, mortality and any toxic reactions. There are no toxic signs or pre-terminal morbidity deaths were observed during the study period. Further, 90 days sub-chronic study was conducted in mice to examine the physical, biochemical, hematological and histopathological observations (Schedule Y). There are no significant toxicological symptoms or any other adverse reactions observed during the sub chronic study in all males and females, it indicates that rhGM-CSF is not toxic up to the $325 \mu \mathrm{g} / 20$ $\mathrm{g}$ mice dose level for mice and $116.6 \mu \mathrm{g} / \mathrm{kg}$ rabbit. Despite the various uses over long time periods, no toxicological data is available regarding the safety of repeated exposure to GM-CSF. Further, the conducting the clinical evaluation will be helpful for establishing the rhGM-CSF as a therapeutic drug in market.

\section{Materials and Methods}

\section{Preparation of test compound}

\section{Test sample}

The expressed rhGM-CSF protein was aseptically formulated rhGM-CSF $(300 \mathrm{mcg} / \mathrm{ml})$ in phosphate buffer, $\mathrm{pH} 7.0$ containing mannitol $(20 \mathrm{mg} / \mathrm{ml})$ and sodium chloride $(150 \mathrm{mM})$ followed by lyophilization and stored at $2-8^{\circ} \mathrm{C}$. The lyophilized powder was reconstituted in injectable water and diluted in $0.9 \%$ normal saline to get the protein solution of required concentration.

\section{Experimental animals}

Both sexes of mice (Mus Musculus) were used for the acute and sub chronic toxicology studies. Healthy pathogenic free, Swiss albino mice weighing 18-25g, 4-6 weeks old and both sexes of New Zealand White rabbits weighing 1.0 -1.8 Kg (Approval No. BT/BS/1753/2001 PID \& BT/BS/1754/2001 PID) were purchased from National Institute of Nutrition (National Center For Laboratory Animal Science, CPCSEA Registered No. 154/1999/CPCSEA Hyderabad, India). All the animals were housed in polypropylene cages in pathogen-free experimental rooms, and were maintained at $22 \pm 3{ }^{\circ} \mathrm{C}$ and relative humidity of $55 \pm 6 \%$ with $12 \mathrm{~h}$ light/dark cycle. During acclimatization, the animals were housed in polycarbonate cages with a standard pellet diet and tap water ad libitum and with $10-15$ air changes per hour in experimental room. All the animals were acclimatized at experimental conditions for 7 days. All experiments were performed in accordance with the Institutional Animal Ethics Committee.

\section{Pre-testing with rhGM-CSF}

A short term study was conducted in ten mice (five males and five females) by single dose administration of rhGMCSF (10 X therapeutic dose, $65 \mu \mathrm{g} / 20 \mathrm{~g}$ mice) intramuscularly (Table 1) at the fore limb and hind limb region of mice on both sides alternatively to evaluate the sensitization of the animal to the test compound. The vehicle control group received $0.9 \%$ normal saline without the test compound. Similarly, single administration of $116.6 \mu \mathrm{g}$ rhGM-CSF per kg rabbit was given intramuscularly. The vehicle control for this study was made by using $0.9 \%$ normal saline without the test compound. All the animals were observed for 15 days for pre-terminal morbidity, mortality and any toxic reactions. 


\section{Sub chronic toxicity study}

Sub-chronic toxicity test was performed by conducting in 80 mice (40 males +40 females) by administering rhGM-CSF daily for thirty days and observed up to 90 days (Griffths and Lumley, 1997). Both sexes of mice were equally divided into four groups with twenty animals each (10 males and 10 females) viz., Group 1: Vehicle control, Group 2: Therapeutic Dose: $32.5 \mu \mathrm{g} / 20$ g mice, Group 3: Intermediate Dose: $162.5 \mu \mathrm{g} / 20 \mathrm{~g}$ mice, Group 4: High Dose: $325 \mu \mathrm{g} / 20 \mathrm{~g}$ mice. The vehicle control group received for this study was made by using $0.9 \%$ normal saline without the test compound. For rabbit, Group 1: Vehicle control, Group 2: Therapeutic Dose: $11.66 \mu \mathrm{g} / \mathrm{kg}$ rabbit, Group 3: Intermediate Dose: $58.3 \mu \mathrm{g} / \mathrm{kg}$ rabbit, Group 4: High dose: $116.6 \mu \mathrm{g} / \mathrm{kg}$ rabbit. The site of injection was cleaned with alcohol swab before and after administering test compound. The test compound as per dosage schedule (Table 1) was administered intramuscularly in the fore limb and hind limb region of mice on both sides alternatively. Sterile disposable syringes of $1 \mathrm{ml}$ (with $1 / 40$ graduation) with 30 gauge needles (1/2 inch) were used for injection. The volume of injection did not exceed more than $200 \mu \mathrm{l}$. The animals were observed for pre-terminal morbidity, mortality and behavioral throughout experimental period. After the experimental period, the mice were fasted overnight (water allowed) blood samples were collected for hematology and clinical observations after which euthanized by $\mathrm{CO}_{2}$ chamber and subjected to gross necropsy and the findings recorded. The individual organs were again examined for gross morphology changes after removal. They were examined clinically and blood was collected for clinical chemistry and hematology after anesthetization which was carried out after $48^{\text {th }} \mathrm{h}$ and $30^{\text {th }}$ day, $60^{\text {th }}$ day and $90^{\text {th }}$ day after the exposure to the test compound. After gross necropsy, organ weights (GM 6101, Sartorious) as well as histopathological examination of various organs such as liver, spleen, kidneys, brain, thymus and injection site, was carried out on day $90^{\text {th }}$ after the last exposure to the test compound. The tissues were sliced adequately wherever necessary. After a minimum of $24 \mathrm{~h}$ fixation, they were sampled and processed by conventional methods, paraffin blocks were made and $6 \mu \mathrm{m}$ paraffin sections were stained with Hematoxylin and Eosin. They were examined under a light microscope. All deviations from normal histology were recorded and compared with corresponding controls. Also, the potential production of neutralizing antibodies was studied.
All the test systems were observed for mortality, live phase of animals (Food intake, body weight, general behavior, Water intake), Cage side observation (Home cage activity, Feces excretion, Urine excretion), Physical examination (Hair Coat, Piloerection, lacrimation, Salivation, Respiration rate and Character, Eye prominence and Eyelid colosure, Convulsion, biting, tremors), Neurological examination (Locomotor activity, rearing activity, tail elevation, abnormal gait, Head position, Pinna touch response), qualitative Urine examination (Urobilinogen, Protein, $\mathrm{P}^{\mathrm{H}}$, RBC, Gravity, ketone, Bilirubin, Gluose) were qualitatively tested using Ames Multistix reagent strips at the time intervals, before exposure to test compound and Post Exposure after the exposure to the test compound (Urine collection was done for 12 hours before testing). Clinical chemistry (glucose, creatinine, total protein, ALP, SGOT, SGPT, bilirubin, potassium, magnesium and calcium) were estimated using Alfa Biotech PLD 951 auto-analyzer, supplied by Wipro Biomed. All analytical kits were purchased from Recombigen Laboratories Pvt Ltd, New Delhi. Hematology parameters (WBC, RBC, Hgb, HCT, MCV, $\mathrm{MCH}, \mathrm{MCHC}$, Platelet Count, and MPV) were estimated by using Beckman Coulter Counter.

\section{Statistical analysis}

Results are expressed as Mean \pm standard error mean (SEM). Data obtained was analyzed by using one way ANOVA followed by Dunnett's test and $p<0.05$ was considered as statistically significant. Treatment groups were compared with vehicle control by fisher's exact or Chi-square test. Between group comparisons by means of Kruskal-Wallis one-way ANOVA and individual group comparisons by Mann-Whitney U test (test groups with vehicle control) were the method of analysis. Heterogeneity of variance was tested by Levene's statistic. After confirming homogeneity, ANOVA linear models were done for between group significant F-ratio. Post-hoc tests by means of Tukey's HSD were carried out for comparison of test groups with vehicle control. Statistical analysis was carried out on SAS 8.2 version.

\section{Results and Discussion}

\section{Pre-testing with rhGMCSF (Acute toxicity study)}

Pre-testing was conducted with the rhGMCSF (10X) alone administered topically using ten mice $(5 \mathrm{M}+5 \mathrm{~F})$ showed no toxic signs or pre-terminal morbidity deaths during the study period. The $\mathrm{LD}_{50}$ for the mice was found to be 
greater than $325 \mu \mathrm{g}$ and greater than $116.6 \mu \mathrm{g}$ for rabbit respectively.

\section{Sub chronic toxicity study}

There were no treatment related toxicity signs and mortality observed in both sexes of mice treated at 32.5 $\mu \mathrm{g} / 20 \mathrm{~g}$ mice, $162.5 \mu \mathrm{g} / 20 \mathrm{~g}$ mice and $325 \mu \mathrm{g} / 20 \mathrm{~g}$ mice intramuscularly during the 90 days observation period. Similarly, there were no treatment related toxicity signs and mortality observed in both sexes of rabbit $11.66 \mu \mathrm{g} / \mathrm{kg}$ rabbit $58.3 \mu \mathrm{g} / \mathrm{kg}$ rabbit $116.6 \mu \mathrm{g} / \mathrm{kg}$ rabbit treated intramuscularly during the 90 days observation period. There is no mortality and morbidity in all the groups during the experimental period. The water and food intake was also not affected by the administration of rhGMCSF and these were similar when compared to vehicle control group animals. The food intake by mice at the initial stages was between 3-5 g and it increased to 4-6 $\mathrm{g}$ on an average in all the groups of animals at the end of the study period (Data was not shown) There were no abnormalities in the head and limb positions, gait, locomotor activity, rearing activity, tail elevations, pinna touch response, fecal consistency and urine color and amount, behavior, activeness, piloerection, lacrimation, salivation, respiration rate, eye prominance, eye lid closure in all treated group animals as wells as vehicle control group animals during the experimental period. There is no convulsions, aggressiveness, biting character and tremors observed in all the groups.

\section{Body weight}

The gain in body weight was normal (10-12 g mice and $1.5 \mathrm{~kg}$ rabbit) in all groups of animals during the study period. There is no significant $(\mathrm{p} \leq 0.05)$ treatment effect on the body weights of male and female mice exposed to test compound at various levels was seen as compared to vehicle control group.

\section{Clinical chemistry}

There were no abnormalities in clinical chemistry parameters viz blood glucose, total protein, SGOT, SGPT, ALP, total bilirubin, creatinine and electrolytes (Magnesium, Potassium and Calcium) in mice treated with rhGMCSF at $48^{\text {th }}$ hour, 30,60 and $90^{\text {th }}$ days when compared with vehicle control group animals. All the parameters observed in vehicle control group animals as well as treated animals were within the physiological range (Table 2). Further, there were no significant differences in the hematological parameters in all treated animals (Table 5).

\section{Hematology}

There were no anomalies observed in hematology parameters including WBC, RBC, platelets, haemoglobin, HCT, MCV, MCH and MCHC at $48^{\text {th }}$ hour, 30, 60 and $90^{\text {th }}$ days in all rhGMCSF treated groups $(\mathrm{p} \leq 0.05)$ comparing with respective vehicle controls (Table $3 \& 4$ ).

\section{Urine analysis}

The $\mathrm{pH}$, specific gravity, glucose, protein, bilirubin, urobilnogen and ketone levels were not affected at $48^{\text {th }}$ hour, $30^{\text {th }}$ day, $60^{\text {th }}$ day and $90^{\text {th }}$ day qualitatively in mice treated with rhGMCSF when compared with vehicle control group animals.

\section{Organ weights}

There were no gross changes in all the organs in all treated group animals and also no significant differences between various organ weights including kidney, liver, spleen and brain collected from all the treated animals with $\operatorname{rhGMCSF}(\mathrm{p} \leq 0.05)$ (Table 5).

\section{Histopathology}

There were no histopathological changes in all the major organs examined, liver, spleen, kidneys, brain, thymus observed in all the test groups as compared to vehicle control group (Data was not shown).

The rhGMCSF has been used for the treatment of malignancies in patients since the rhGMCSF is able to shorten the time period of neutropenia after chemotherapy and to reduce neutropenia-related morbidity such as infections (Schulz et al., 1991). Further it has many applications while using as therapeutic drug for many diseases such as acute myeloid leukemia (Lanza et al., 1997), solid tumors (de Carren et al., 1997), lung cancer (Gautier et al., 1995).

Since, rhGMCSF has vast applications in treatment of various diseases and cost effective, the recombinant GMCSF has been developed in E.coli which has shown similar properties with the natural sources (Chitra et al., 2017) and further the pre-clinical toxicology study was conducted before trail the clinical applications. 
Table.1 rhGMCSF administration to mice in acute and sub-chronic toxicity study

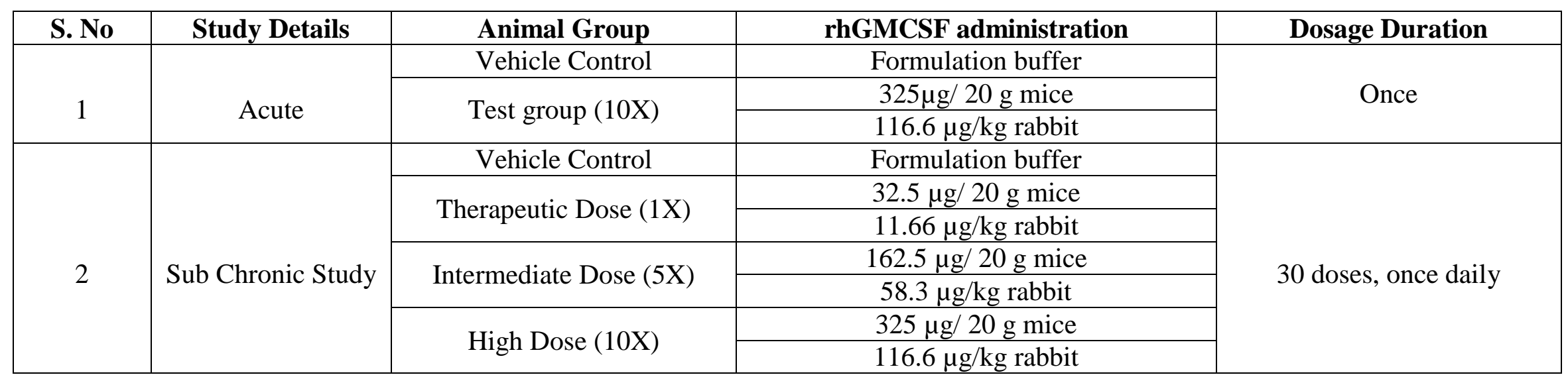

Table.6 Rabbit organ weights at 90th day

\begin{tabular}{|c|c|c|c|c|c|c|c|c|}
\hline & \multicolumn{4}{|c|}{ Rabbit } & \multicolumn{4}{|c|}{ Mice } \\
\hline \multirow[t]{2}{*}{ Parameters } & \multicolumn{4}{|c|}{ Groups (Average of 10 Rabbits) } & \multicolumn{4}{|c|}{ Groups (Average of 20 Mice ) } \\
\hline & VC & TD & ID & HD & VC & TD & ID & HD \\
\hline Spleen & $0.86 \pm 0.09$ & $0.88 \pm 0.10$ & $0.96 \pm 0.08$ & $0.96 \pm 0.08$ & $0.110 \pm 0.044$ & $0.100 \pm 0.00$ & $0.100 \pm 0.00$ & $0.100 \pm 0.00$ \\
\hline Heart & $4.06 \pm 0.09$ & $4.05 \pm 0.15$ & $4.00 \pm 0.00$ & $4.05 \pm 0.15$ & $0.100 \pm 0.00$ & $0.100 \pm 0.00$ & $0.100 \pm 0.00$ & $0.100 \pm 0.00$ \\
\hline Kidney & $11.08 \pm 0.87$ & $12.55 \pm 0.59$ & $13.20 \pm 0.63$ & $13.32 \pm 0.96$ & $0.42 \pm 0.052$ & $0.415 \pm 0.048$ & $0.420 \pm 0.52$ & $0.375 \pm 0.055$ \\
\hline Brain & $6.49 \pm 0.17$ & $6.35 \pm 0.33$ & $6.090 \pm 0.16$ & $6.345 \pm 0.32$ & $0.375 \pm 0.044$ & $0.350 \pm 0.051$ & $0.390 \pm 0044$ & $0.375 \pm 0.044$ \\
\hline
\end{tabular}


Int.J.Curr.Res.Aca.Rev.2017; 5(6): 28-37

Table.2 Sub acute toxicity study - Clinical chemistry parameters in mice administered with rhGMCSF

\begin{tabular}{|c|c|c|c|c|c|c|c|c|c|c|c|c|c|c|c|c|}
\hline \multirow{2}{*}{\begin{tabular}{c|} 
Parameter \\
Duration
\end{tabular}} & \multicolumn{4}{|c|}{ Vehicle control } & \multicolumn{4}{|c|}{ Therapeutic Dose } & \multicolumn{4}{|c|}{ Intermediate Dose } & \multicolumn{4}{|c|}{ High Dose } \\
\hline & $48 \mathrm{~h}$ & $30 \mathrm{~d}$ & $60 \mathrm{~d}$ & $90 \mathrm{~d}$ & $48 \mathrm{~h}$ & $30 \mathrm{~d}$ & $60 \mathrm{~d}$ & $90 \mathrm{~d}$ & $48 \mathrm{~h}$ & $30 \mathrm{~d}$ & $60 \mathrm{~d}$ & $90 \mathrm{~d}$ & $48 \mathrm{~h}$ & $30 \mathrm{~d}$ & $60 \mathrm{~d}$ & $90 \mathrm{~d}$ \\
\hline $\begin{array}{c}\text { Blood } \\
\text { Glucose } \\
\text { (mg/dl) }\end{array}$ & $\begin{array}{c}67.20 \\
\pm \\
3.28\end{array}$ & $\begin{array}{c}71.52 \\
\pm \\
7.97\end{array}$ & $\begin{array}{c}78.54 \\
\pm \\
14.0\end{array}$ & $\begin{array}{c}71.27 \\
\pm \\
6.50\end{array}$ & $\begin{array}{c}70.98 \\
\pm \\
2.44\end{array}$ & $\begin{array}{c}77.75 \\
\pm \\
10.03\end{array}$ & $\begin{array}{c}76.76 \\
\pm \\
12.5\end{array}$ & $\begin{array}{c}74.86 \\
\pm \\
6.13\end{array}$ & $\begin{array}{c}69.64 \\
\pm \\
4.40\end{array}$ & $\begin{array}{c}81.83 \\
\pm \\
9.92\end{array}$ & $\begin{array}{c}79.65 \\
\pm \\
9.15\end{array}$ & $\begin{array}{c}77.39 \\
\pm \\
6.34\end{array}$ & $\begin{array}{c}71.45 \\
\pm \\
3.31\end{array}$ & $\begin{array}{c}82.18 \\
\pm \\
9.05\end{array}$ & $\begin{array}{c}80.52 \\
\pm \\
1.97\end{array}$ & $\begin{array}{c}77.72 \\
\pm \\
6.93\end{array}$ \\
\hline $\begin{array}{c}\text { Total } \\
\text { Protein } \\
(\mathrm{gm} / \mathrm{dl})\end{array}$ & $\begin{array}{c}2.16 \\
\pm \\
0.15\end{array}$ & $\begin{array}{c}2.24 \\
\pm \\
0.08\end{array}$ & $\begin{array}{c}2.34 \\
\pm \\
0.25\end{array}$ & $\begin{array}{c}2.25 \\
\pm \\
0.11\end{array}$ & $\begin{array}{c}2.27 \\
\pm \\
0.14\end{array}$ & $\begin{array}{c}2.18 \\
\pm \\
0.16\end{array}$ & $\begin{array}{c}2.43 \\
\pm \\
0.23\end{array}$ & $\begin{array}{c}2.28 \\
\pm \\
0.19\end{array}$ & $\begin{array}{c}2.27 \\
\pm \\
0.26\end{array}$ & $\begin{array}{c}2.30 \\
\pm \\
0.17\end{array}$ & $\begin{array}{c}2.21 \\
\pm \\
0.15\end{array}$ & $\begin{array}{c}2.25 \\
\pm \\
0.20\end{array}$ & $\begin{array}{c}2.29 \\
\pm \\
0.20\end{array}$ & $\begin{array}{c}2.37 \\
\pm \\
0.15\end{array}$ & $\begin{array}{c}2.26 \\
\pm \\
0.23\end{array}$ & $\begin{array}{c}2.25 \\
\pm \\
0.20\end{array}$ \\
\hline $\begin{array}{l}\text { SGOT } \\
\text { (IU/L) }\end{array}$ & $\begin{array}{c}33.34 \\
\pm \\
5.43\end{array}$ & $\begin{array}{c}33.60 \\
\pm \\
3.39\end{array}$ & $\begin{array}{c}32.66 \\
\pm \\
4.70\end{array}$ & $\begin{array}{c}36.14 \\
\pm \\
2.55\end{array}$ & $\begin{array}{c}32.51 \\
\pm \\
5.0\end{array}$ & $\begin{array}{c}34.71 \\
\pm \\
5.08\end{array}$ & $\begin{array}{c}34.45 \\
\pm \\
3.31\end{array}$ & $\begin{array}{c}36.72 \\
\pm \\
4.31\end{array}$ & $\begin{array}{c}35.68 \\
\pm \\
5.58\end{array}$ & $\begin{array}{c}34.95 \\
\pm \\
2.64\end{array}$ & $\begin{array}{c}34.30 \\
\pm \\
2.88\end{array}$ & $\begin{array}{c}36.24 \\
\pm \\
3.22\end{array}$ & $\begin{array}{c}36.16 \\
\pm \\
2.65\end{array}$ & $\begin{array}{c}34.93 \\
\pm \\
3.48\end{array}$ & $\begin{array}{c}35.97 \\
\pm \\
2.27\end{array}$ & $\begin{array}{c}35.30 \\
\pm \\
3.38\end{array}$ \\
\hline $\begin{array}{l}\text { SGPT } \\
\text { (IU/L) }\end{array}$ & $\begin{array}{c}21.84 \\
\pm \\
3.61\end{array}$ & $\begin{array}{c}21.38 \\
\pm \\
4.05\end{array}$ & $\begin{array}{c}21.53 \\
\pm \\
3.53\end{array}$ & $\begin{array}{c}23.79 \\
\pm \\
3.77\end{array}$ & $\begin{array}{c}21.63 \\
\pm \\
3.84\end{array}$ & $\begin{array}{c}23.46 \\
\pm \\
6.82\end{array}$ & $\begin{array}{c}23.01 \\
\pm \\
3.72\end{array}$ & $\begin{array}{c}25.50 \\
\pm \\
3.76\end{array}$ & $\begin{array}{c}24.31 \\
\pm \\
4.02\end{array}$ & $\begin{array}{c}24.77 \\
\pm \\
6.73\end{array}$ & $\begin{array}{c}23.21 \\
\pm \\
4.54\end{array}$ & $\begin{array}{c}24.22 \\
\pm \\
5.21\end{array}$ & $\begin{array}{c}24.18 \\
\pm \\
4.54\end{array}$ & $\begin{array}{c}24.53 \\
\pm \\
5.57\end{array}$ & $\begin{array}{c}23.58 \\
\pm \\
3.23\end{array}$ & $\begin{array}{c}23.96 \\
\pm \\
3.17\end{array}$ \\
\hline $\begin{array}{c}\text { ALP } \\
\text { (IU/L) }\end{array}$ & $\begin{array}{c}40.14 \\
\pm \\
5.83\end{array}$ & $\begin{array}{c}42.17 \\
\pm \\
6.42\end{array}$ & $\begin{array}{c}39.38 \\
\pm \\
4.39\end{array}$ & $\begin{array}{c}40.54 \\
\pm \\
3.66\end{array}$ & $\begin{array}{c}37.60 \\
\pm \\
5.10\end{array}$ & $\begin{array}{c}42.85 \\
\pm \\
6.00\end{array}$ & $\begin{array}{c}40.22 \\
\pm \\
4.48\end{array}$ & $\begin{array}{c}40.58 \\
\pm \\
4.12\end{array}$ & $\begin{array}{c}36.46 \\
\pm \\
2.94\end{array}$ & $\begin{array}{c}39.09 \\
\pm \\
5.50\end{array}$ & $\begin{array}{c}39.06 \\
\pm \\
4.51\end{array}$ & $\begin{array}{c}39.96 \\
\pm \\
3.17\end{array}$ & $\begin{array}{c}34.37 \\
\pm \\
3.58\end{array}$ & $\begin{array}{c}36.18 \\
\pm \\
3.25\end{array}$ & $\begin{array}{c}38.31 \\
\pm \\
2.81\end{array}$ & $\begin{array}{c}40.02 \\
\pm \\
2.85\end{array}$ \\
\hline $\begin{array}{c}\text { Total } \\
\text { bilirubin } \\
(\mathrm{mg} / \mathrm{dl})\end{array}$ & $\begin{array}{c}0.39 \\
\pm \\
0.28 \\
\end{array}$ & $\begin{array}{c}0.35 \\
\pm \\
0.28 \\
\end{array}$ & $\begin{array}{c}0.350 \\
\pm \\
0.31 \\
\end{array}$ & $\begin{array}{c}0.329 \\
\pm \\
0.19 \\
\end{array}$ & $\begin{array}{c}0.35 \\
\pm \\
0.17 \\
\end{array}$ & $\begin{array}{c}0.53 \\
\pm \\
0.30 \\
\end{array}$ & $\begin{array}{c}0.37 \\
\pm \\
0.22 \\
\end{array}$ & $\begin{array}{c}0.36 \\
\pm \\
0.14 \\
\end{array}$ & $\begin{array}{c}0.29 \\
\pm \\
0.09 \\
\end{array}$ & $\begin{array}{c}0.43 \\
\pm \\
0.19 \\
\end{array}$ & $\begin{array}{c}0.361 \\
\pm \\
0.25 \\
\end{array}$ & $\begin{array}{c}0.39 \\
\pm \\
0.18 \\
\end{array}$ & $\begin{array}{c}0.31 \\
\pm \\
0.14 \\
\end{array}$ & $\begin{array}{c}0.370 \\
\pm \\
0.14 \\
\end{array}$ & $\begin{array}{c}0.388 \\
\pm \\
0.17 \\
\end{array}$ & $\begin{array}{c}0.41 \\
\pm \\
0.16 \\
\end{array}$ \\
\hline Trigly & $\begin{array}{c}92.19 \\
\pm \\
10.7 \\
\end{array}$ & $\begin{array}{c}91.58 \\
\pm \\
8.70 \\
\end{array}$ & $\begin{array}{c}90.85 \\
\pm \\
8.62 \\
\end{array}$ & $\begin{array}{c}92.74 \\
\pm \\
10.10 \\
\end{array}$ & $\begin{array}{c}91.57 \\
\pm \\
9.38 \\
\end{array}$ & $\begin{array}{c}92.34 \\
\pm \\
12.44 \\
\end{array}$ & $\begin{array}{c}90.51 \\
\pm \\
11.07 \\
\end{array}$ & $\begin{array}{c}93.17 \\
\pm \\
10.16 \\
\end{array}$ & $\begin{array}{c}90.28 \\
\pm \\
8.78 \\
\end{array}$ & $\begin{array}{c}91.73 \\
\pm \\
10.72 \\
\end{array}$ & $\begin{array}{c}91.29 \\
\pm \\
10.18 \\
\end{array}$ & $\begin{array}{c}94.28 \\
\pm \\
11.09 \\
\end{array}$ & $\begin{array}{c}90.87 \\
\pm \\
11.54 \\
\end{array}$ & $\begin{array}{c}90.20 \\
\pm \\
12.0 \\
\end{array}$ & $\begin{array}{c}92.90 \\
\pm \\
8.99 \\
\end{array}$ & $\begin{array}{c}94.12 \\
\pm \\
9.00 \\
\end{array}$ \\
\hline Cholesterol & $\begin{array}{c}77.93 \\
\pm \\
9.01\end{array}$ & $\begin{array}{c}79.28 \\
\pm \\
9.28\end{array}$ & $\begin{array}{c}75.86 \\
\pm \\
7.50\end{array}$ & $\begin{array}{c}75.53 \\
\pm \\
7.22\end{array}$ & $\begin{array}{c}79.50 \\
\pm \\
8.20\end{array}$ & $\begin{array}{c}76.33 \\
\pm \\
5.23\end{array}$ & $\begin{array}{c}72.22 \\
\pm \\
5.24\end{array}$ & $\begin{array}{c}74.49 \\
\pm \\
4.41\end{array}$ & $\begin{array}{c}77.60 \\
\pm \\
6.78\end{array}$ & $\begin{array}{c}74.74 \\
\pm \\
6.53\end{array}$ & $\begin{array}{c}72.47 \\
\pm \\
3.08\end{array}$ & $\begin{array}{c}74.97 \\
\pm \\
7.21\end{array}$ & $\begin{array}{c}73.51 \\
\pm \\
8.27\end{array}$ & $\begin{array}{c}72.21 \\
\pm \\
7.64\end{array}$ & $\begin{array}{c}70.26 \\
\pm \\
6.97\end{array}$ & $\begin{array}{c}70.56 \\
\pm \\
6.14\end{array}$ \\
\hline $\begin{array}{c}\text { Magnesium } \\
(\mathrm{m.mol} / \mathrm{L})\end{array}$ & $\begin{array}{c}1.742 \\
\pm \\
0.247 \\
\end{array}$ & $\begin{array}{c}1.56 \\
\pm \\
0.311 \\
\end{array}$ & $\begin{array}{c}1.69 \\
\pm \\
0.26 \\
\end{array}$ & $\begin{array}{c}1.570 \\
\pm \\
0.219 \\
\end{array}$ & $\begin{array}{c}1.74 \\
\pm \\
0.102 \\
\end{array}$ & $\begin{array}{c}1.50 \\
\pm \\
0.370 \\
\end{array}$ & $\begin{array}{c}1.81 \\
\pm \\
0.102 \\
\end{array}$ & $\begin{array}{c}1.729 \\
\pm \\
0.237 \\
\end{array}$ & $\begin{array}{c}1.76 \\
\pm \\
0.064 \\
\end{array}$ & $\begin{array}{c}1.465 \\
\pm \\
0.445 \\
\end{array}$ & $\begin{array}{c}1.800 \\
\pm \\
0.056 \\
\end{array}$ & $\begin{array}{c}1.748 \\
\pm \\
0.150 \\
\end{array}$ & $\begin{array}{c}1.804 \\
\pm \\
0.04 \\
\end{array}$ & $\begin{array}{c}1.55 \\
\pm \\
0.332 \\
\end{array}$ & $\begin{array}{c}1.688 \\
\pm \\
0.29 \\
\end{array}$ & $\begin{array}{c}1.818 \\
\pm \\
0.076 \\
\end{array}$ \\
\hline $\begin{array}{c}\text { Potassium } \\
(\mathrm{m} . \mathrm{mol} / \mathrm{L})\end{array}$ & $\begin{array}{c}5.02 \\
\pm \\
0.603\end{array}$ & $\begin{array}{c}4.96 \\
\pm \\
0.640\end{array}$ & $\begin{array}{c}4.98 \\
\pm \\
0.873\end{array}$ & $\begin{array}{c}5.10 \\
\pm \\
0.908\end{array}$ & $\begin{array}{c}5.134 \\
\pm \\
0.53\end{array}$ & $\begin{array}{c}5.105 \\
\pm \\
0.692\end{array}$ & $\begin{array}{c}5.29 \\
\pm \\
0.731\end{array}$ & $\begin{array}{c}5.40 \\
\pm \\
0.61\end{array}$ & $\begin{array}{c}5.00 \\
\pm \\
0.772\end{array}$ & $\begin{array}{c}4.96 \\
\pm \\
0.691\end{array}$ & $\begin{array}{c}5.04 \\
\pm \\
0.928\end{array}$ & $\begin{array}{c}5.177 \\
\pm \\
0.416\end{array}$ & $\begin{array}{c}5.05 \\
\pm \\
0.577\end{array}$ & $\begin{array}{c}5.114 \\
\pm \\
0.788\end{array}$ & $\begin{array}{c}5.22 \\
\pm \\
1.04\end{array}$ & $\begin{array}{c}5.05 \\
\pm \\
0.957\end{array}$ \\
\hline $\begin{array}{c}\text { Urea } \\
(\mathrm{mg} / \mathrm{dl})\end{array}$ & $\begin{array}{c}14.23 \\
\pm \\
2.09\end{array}$ & $\begin{array}{c}16.26 \\
\pm \\
1.15\end{array}$ & $\begin{array}{c}15.45 \\
\pm \\
2.83\end{array}$ & $\begin{array}{c}15.31 \\
\pm \\
2.61\end{array}$ & $\begin{array}{c}13.25 \\
\pm \\
1.73\end{array}$ & $\begin{array}{c}14.85 \\
\pm \\
2.32\end{array}$ & $\begin{array}{c}16.70 \\
\pm \\
3.05\end{array}$ & $\begin{array}{c}16.90 \\
\pm \\
2.93\end{array}$ & $\begin{array}{c}12.57 \\
\pm \\
3.04\end{array}$ & $\begin{array}{c}14.88 \\
\pm \\
1.95\end{array}$ & $\begin{array}{c}17.80 \\
\pm \\
3.85\end{array}$ & $\begin{array}{c}17.59 \\
\pm \\
4.55\end{array}$ & $\begin{array}{c}11.44 \\
\pm \\
1.50\end{array}$ & $\begin{array}{c}16.74 \\
\pm \\
2.50\end{array}$ & $\begin{array}{c}16.95 \\
\pm \\
2.37\end{array}$ & $\begin{array}{c}17.00 \\
\pm \\
1.31\end{array}$ \\
\hline
\end{tabular}


Int.J.Curr.Res.Aca.Rev.2017; 5(6): 28-37

Table.3 Hematological report of Mice for WBC, RBC, Hgb and HCT (\%)

\begin{tabular}{|c|c|c|c|c|c|c|c|c|c|c|c|c|c|c|c|c|}
\hline Days & \multicolumn{4}{|c|}{ Vehicle control } & \multicolumn{4}{|c|}{ Therapeutic Dose } & \multicolumn{4}{|c|}{ Intermediate Dose } & \multicolumn{4}{|c|}{ High Dose } \\
\hline $\begin{array}{c}\text { Param } \\
\text { eters }\end{array}$ & $\begin{array}{c}\text { WBC } \\
(\mathrm{x} \\
03 \mu \mathrm{l})\end{array}$ & $\begin{array}{c}\mathrm{RBC} \\
(\mathrm{x} \\
\left.0^{6} \mu \mathrm{l}\right)\end{array}$ & $\begin{array}{l}\mathrm{Hgb} \\
(\mathrm{g} / \mathrm{dl})\end{array}$ & $\begin{array}{c}\mathrm{HCT} \\
(\%)\end{array}$ & $\begin{array}{c}\text { WBC } \\
(\mathrm{x} \\
03 \mu \mathrm{l})\end{array}$ & $\begin{array}{c}\text { RBC } \\
(\mathrm{x} \\
\left.0^{6} \mu 1\right)\end{array}$ & $\begin{array}{c}\mathrm{Hgb} \\
(\mathrm{g} / \mathrm{dl})\end{array}$ & $\begin{array}{c}\mathrm{HCT} \\
(\%)\end{array}$ & $\begin{array}{c}\text { WBC } \\
(x \\
03 \mu l)\end{array}$ & $\begin{array}{c}\text { RBC } \\
(\mathrm{x} \\
\left.0^{6} \mu \mathrm{l}\right)\end{array}$ & $\begin{array}{l}\mathrm{Hgb} \\
(\mathrm{g} / \mathrm{dl})\end{array}$ & $\begin{array}{l}\mathrm{HCT} \\
(\%)\end{array}$ & $\begin{array}{c}\text { WBC } \\
(\mathrm{x} \\
03 \mu \mathrm{l})\end{array}$ & $\begin{array}{c}\text { RBC } \\
(\mathrm{x} \\
\left.0^{6} \mu \mathrm{l}\right)\end{array}$ & $\begin{array}{c}\mathrm{Hgb} \\
(\mathrm{g} / \mathrm{dl})\end{array}$ & $\begin{array}{c}\mathrm{HCT} \\
(\%)\end{array}$ \\
\hline $48 \mathrm{Hrs}$ & $\begin{array}{c}11.70 \pm \\
1.36\end{array}$ & $\begin{array}{c}10.06 \pm \\
0.42\end{array}$ & $\begin{array}{c}14.92 \pm \\
1.72\end{array}$ & $\begin{array}{c}46.7 \pm 2 \\
.50\end{array}$ & $\begin{array}{c}10.91 \pm \\
1.72\end{array}$ & $\begin{array}{c}10.11 \pm \\
0.51\end{array}$ & $\begin{array}{c}14.95 \pm \\
1.70\end{array}$ & $\begin{array}{c}47.21 \pm \\
2.65\end{array}$ & $\begin{array}{c}9.87 \pm \\
1.36\end{array}$ & $\begin{array}{c}10.12 \pm \\
0.23\end{array}$ & $\begin{array}{c}15.97 \pm \\
0.38\end{array}$ & $\begin{array}{c}47.88 \pm \\
1.35\end{array}$ & $\begin{array}{c}10.17 \pm \\
1.92\end{array}$ & $\begin{array}{c}10.69 \pm \\
1.85\end{array}$ & $\begin{array}{c}15.3 \pm 1 \\
.62\end{array}$ & $\begin{array}{c}44.47 \pm \\
10.17\end{array}$ \\
\hline 60 day & $\begin{array}{c}8.79 \pm 2 \\
.19\end{array}$ & $\begin{array}{c}10.04 \pm \\
0.56\end{array}$ & $\begin{array}{c}15.85 \pm \\
0.84\end{array}$ & $\begin{array}{c}47.4 \pm \\
2.62\end{array}$ & $\begin{array}{c}8.01 \pm 2 \\
.84\end{array}$ & $\begin{array}{c}10.12 \pm \\
0.24\end{array}$ & $\begin{array}{c}15.09 \pm \\
2.35\end{array}$ & $\begin{array}{c}48.12 \pm \\
2.42\end{array}$ & $\begin{array}{c}6.36 \pm \\
1.38\end{array}$ & $\begin{array}{c}10.61 \pm \\
0.41\end{array}$ & $\begin{array}{c}16.78 \pm \\
0.72\end{array}$ & $\begin{array}{c}50.06 \pm \\
2.00\end{array}$ & $\begin{array}{c}7.64 \pm 2 \\
.36\end{array}$ & $\begin{array}{c}10.39 \pm \\
0.54\end{array}$ & $\begin{array}{c}16.5 \pm 0 \\
.67\end{array}$ & $\begin{array}{c}48.52 \pm \\
1.81\end{array}$ \\
\hline 90day & $\begin{array}{c}6.96 \pm 1 \\
.92\end{array}$ & $\begin{array}{c}10.21 \pm \\
0.45\end{array}$ & $\begin{array}{c}16.34 \pm \\
0.76\end{array}$ & $\begin{array}{c}48.39 \pm \\
1.59\end{array}$ & $\begin{array}{c}7.68 \pm 2 \\
.84\end{array}$ & $\begin{array}{c}10.39 \pm \\
0.47\end{array}$ & $\begin{array}{c}16.35 \pm \\
0.69\end{array}$ & $\begin{array}{c}47.99 \pm \\
1.95\end{array}$ & $\begin{array}{c}6.82 \pm \\
2.98\end{array}$ & $\begin{array}{c}10.65 \pm \\
0.80\end{array}$ & $\begin{array}{c}17.47 \pm \\
1.00\end{array}$ & $\begin{array}{c}50.86 \pm \\
3.22\end{array}$ & $\begin{array}{c}6.67 \pm 3 \\
.41\end{array}$ & $\begin{array}{c}10.71 \pm \\
0.41\end{array}$ & $\begin{array}{c}16.97 \pm \\
0.67\end{array}$ & $\begin{array}{c}50.44 \pm \\
2.02\end{array}$ \\
\hline
\end{tabular}

\begin{tabular}{|c|c|c|c|c|c|c|c|c|c|c|c|c|c|c|c|c|}
\hline Days & \multicolumn{4}{|c|}{ Vehicle control } & \multicolumn{4}{|c|}{ Therapeutic Dose } & \multicolumn{4}{|c|}{ Intermediate Dose } & \multicolumn{4}{|c|}{ Days } \\
\hline $\begin{array}{l}\text { Param } \\
\text { eters }\end{array}$ & $\begin{array}{c}\text { WBC( } \\
x \\
03 \mu 1) \\
\end{array}$ & $\begin{array}{c}\text { RBC } \\
(\mathrm{x} \\
\left.0^{6} \mu \mathrm{l}\right) \\
\end{array}$ & $\begin{array}{l}\mathrm{Hgb} \\
(\mathrm{g} / \mathrm{dl})\end{array}$ & $\begin{array}{c}\mathrm{HCT} \\
(\%)\end{array}$ & $\begin{array}{c}\text { WBC } \\
(x \\
03 \mu l) \\
\end{array}$ & $\begin{array}{c}\text { RBC } \\
(\mathrm{x} \\
\left.0^{6} \mu \mathrm{l}\right) \\
\end{array}$ & $\begin{array}{l}\mathrm{Hgb} \\
(\mathrm{g} / \mathrm{dl})\end{array}$ & $\begin{array}{c}\mathrm{HCT} \\
(\%)\end{array}$ & $\begin{array}{c}\text { WBC } \\
(x \\
03 \mu 1) \\
\end{array}$ & $\begin{array}{c}\mathrm{RBC} \\
\left(\mathrm{x} 0^{6}\right. \\
\mu \mathrm{l})\end{array}$ & $\begin{array}{l}\text { Hgb } \\
(\mathrm{g} / \mathrm{dl})\end{array}$ & $\begin{array}{c}\mathrm{HCT} \\
(\%)\end{array}$ & $\begin{array}{c}\text { WBC } \\
(\mathrm{x} \\
03 \mu \mathrm{l}) \\
\end{array}$ & $\begin{array}{c}\text { RBC } \\
(\mathrm{x} \\
\left.0^{6} \mu \mathrm{l}\right) \\
\end{array}$ & $\begin{array}{l}\text { Hgb } \\
(\mathrm{g} / \mathrm{dl})\end{array}$ & $\begin{array}{c}\mathrm{HCT} \\
(\%)\end{array}$ \\
\hline $48 \mathrm{Hrs}$ & $\begin{array}{c}4.24 \pm \\
0.62 \\
\end{array}$ & $\begin{array}{c}15.97 \pm \\
3.16\end{array}$ & $\begin{array}{c}12.82 \pm \\
0.67\end{array}$ & $\begin{array}{c}41.16 \pm \\
0.90\end{array}$ & $\begin{array}{c}4.36 \pm \\
0.20 \\
\end{array}$ & $\begin{array}{c}15.67 \pm \\
1.71 \\
\end{array}$ & $\begin{array}{c}12.56 \pm \\
1.20\end{array}$ & $\begin{array}{c}39.2 \pm 1 \\
.86 \\
\end{array}$ & $\begin{array}{c}6.32 \pm \\
1.30 \\
\end{array}$ & $\begin{array}{c}14.76 \pm \\
1.81\end{array}$ & $\begin{array}{c}12.56 \pm \\
0.55\end{array}$ & $\begin{array}{c}13.30 \pm \\
0.86 \\
\end{array}$ & $\begin{array}{c}4.28 \pm \\
0.32 \\
\end{array}$ & $\begin{array}{c}13.97 \pm \\
1.71 \\
\end{array}$ & $\begin{array}{c}12.40 \pm \\
0.55\end{array}$ & $\begin{array}{c}39.34 \pm \\
1.61 \\
\end{array}$ \\
\hline $\begin{array}{c}30 \\
\text { days }\end{array}$ & $\begin{array}{c}4.34 \pm \\
0.39\end{array}$ & $\begin{array}{c}13.21 \pm \\
1.80\end{array}$ & $\begin{array}{c}12.32 \pm \\
0.57\end{array}$ & $\begin{array}{c}39.20 \pm \\
1.81 \\
\end{array}$ & $\begin{array}{c}4.62 \pm \\
0.29 \\
\end{array}$ & $\begin{array}{c}15.07 \pm \\
2.08 \\
\end{array}$ & $\begin{array}{c}12.34 \pm \\
2.59\end{array}$ & $\begin{array}{c}41.1 \pm 5 \\
.06\end{array}$ & $\begin{array}{c}4.52 \pm \\
0.35 \\
\end{array}$ & $\begin{array}{c}14.19 \pm \\
1.69\end{array}$ & $\begin{array}{c}11.92 \pm \\
0.91 \\
\end{array}$ & $\begin{array}{c}39.50 \pm \\
2.39\end{array}$ & $\begin{array}{c}4.18 \pm \\
0.26 \\
\end{array}$ & $\begin{array}{c}13.74 \pm \\
1.89\end{array}$ & $\begin{array}{c}12.68 \pm \\
1.16\end{array}$ & $\begin{array}{c}40.82 \pm \\
3.90 \\
\end{array}$ \\
\hline 60 day & $\begin{array}{c}3.70 \pm \\
0.33 \\
\end{array}$ & $\begin{array}{c}13.72 \pm \\
1.53\end{array}$ & $\begin{array}{c}12.94 \pm \\
1.57 \\
\end{array}$ & $\begin{array}{c}40.48 \pm \\
3.50\end{array}$ & $\begin{array}{c}4.22 \pm \\
0.23 \\
\end{array}$ & $\begin{array}{c}14.62 \pm \\
1.21 \\
\end{array}$ & $\begin{array}{c}13.16 \pm \\
0.82 \\
\end{array}$ & $\begin{array}{c}41.6 \pm 2 \\
.35 \\
\end{array}$ & $\begin{array}{c}3.98 \pm \\
0.36 \\
\end{array}$ & $\begin{array}{c}13.85 \pm \\
1.30 \\
\end{array}$ & $\begin{array}{c}11.94 \pm \\
0.82 \\
\end{array}$ & $\begin{array}{c}38.72 \pm \\
2.29\end{array}$ & $\begin{array}{c}3.92 \pm \\
0.54 \\
\end{array}$ & $\begin{array}{c}15.09 \pm \\
1.64\end{array}$ & $\begin{array}{c}11.94 \pm \\
0.82 \\
\end{array}$ & $\begin{array}{c}39.48 \pm \\
3.37 \\
\end{array}$ \\
\hline 90day & $\begin{array}{c}4.45 \pm \\
0.36\end{array}$ & $\begin{array}{c}13.81 \pm \\
1.41\end{array}$ & $\begin{array}{c}12.56 \pm \\
1.11\end{array}$ & $\begin{array}{c}40.88 \pm \\
3.45\end{array}$ & $\begin{array}{c}4.36 \pm \\
0.18\end{array}$ & $\begin{array}{c}13.10 \pm \\
1.36\end{array}$ & $\begin{array}{c}13.44 \pm \\
1.32\end{array}$ & $\begin{array}{c}41.58 \pm \\
3.45\end{array}$ & $\begin{array}{c}4.47 \pm \\
0.20\end{array}$ & $\begin{array}{c}13.28 \pm \\
0.95\end{array}$ & $\begin{array}{c}12.56 \pm \\
0.55\end{array}$ & $\begin{array}{c}40.60 \pm \\
2.33\end{array}$ & $\begin{array}{c}4.52 \pm \\
0.40\end{array}$ & $\begin{array}{c}13.92 \pm \\
1.83\end{array}$ & $\begin{array}{c}13.70 \pm \\
0.75\end{array}$ & $\begin{array}{c}42.30 \pm \\
3.27\end{array}$ \\
\hline
\end{tabular}


Int.J.Curr.Res.Aca.Rev.2017; 5(6): 28-37

Table.4 Hematological report of Rabbits for MCV, MCH, MCHC and Plt

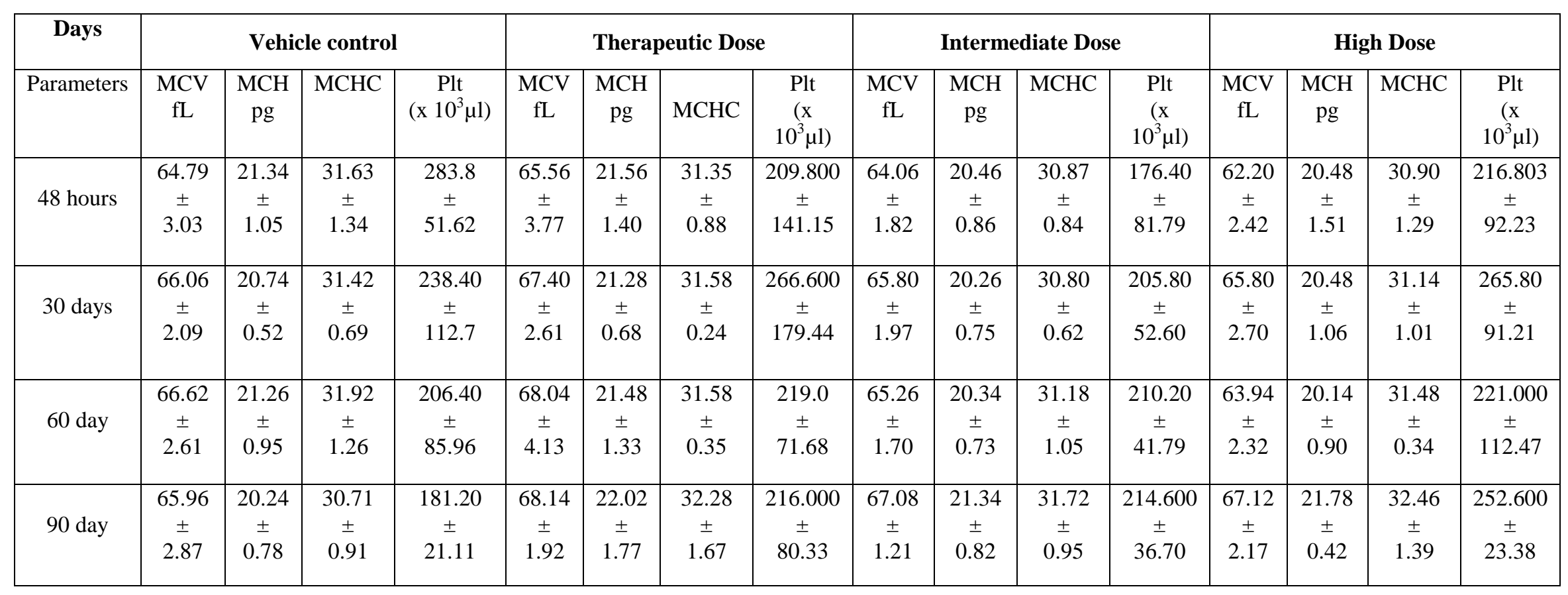

Table.5 Hematological report of mice for MCV, MCH, MCHC and Plt

\begin{tabular}{|c|c|c|c|c|c|c|c|c|c|c|c|c|c|c|c|c|}
\hline Days & \multicolumn{4}{|c|}{ Vehicle control } & \multicolumn{4}{|c|}{ Therapeutic Dose } & \multicolumn{4}{|c|}{ Intermediate Dose } & \multicolumn{4}{|c|}{ High Dose } \\
\hline $\begin{array}{c}\text { Para } \\
\text { meter } \\
\end{array}$ & $\begin{array}{c}\mathrm{MCV} \\
\mathrm{fL}\end{array}$ & $\begin{array}{c}\mathrm{MCH} \\
\mathrm{pg}\end{array}$ & $\begin{array}{c}\mathrm{MCH} \\
\mathrm{C}\end{array}$ & $\begin{array}{c}\text { Plt } \\
(x \\
103 س)\end{array}$ & $\begin{array}{c}\mathrm{MCV} \\
\mathrm{fL}\end{array}$ & $\begin{array}{c}\mathrm{MCH} \\
\mathrm{pg}\end{array}$ & $\begin{array}{c}\mathrm{MCH} \\
\mathrm{C}\end{array}$ & $\begin{array}{c}\text { Plt } \\
(\mathrm{x} \\
103 \mathrm{w}) \\
\end{array}$ & $\begin{array}{c}\mathrm{MCV} \\
\mathrm{fL}\end{array}$ & $\begin{array}{c}\mathrm{MCH} \\
\mathrm{pg}\end{array}$ & $\begin{array}{c}\mathrm{MCH} \\
\mathrm{C}\end{array}$ & $\begin{array}{c}\text { Plt } \\
(x \\
1031)\end{array}$ & $\begin{array}{c}\mathrm{MCV} \\
\mathrm{fL}\end{array}$ & $\begin{array}{c}\mathrm{MCH} \\
\mathrm{pg}\end{array}$ & $\begin{array}{c}\mathrm{MCH} \\
\mathrm{C}\end{array}$ & $\begin{array}{c}\text { Plt } \\
(x \\
103 w 1)\end{array}$ \\
\hline $\begin{array}{c}48 \\
\text { hours }\end{array}$ & $\begin{array}{l}46.41 \\
\pm 1.26\end{array}$ & $\begin{array}{l}15.41 \\
\pm 0.38\end{array}$ & $\begin{array}{l}33.21 \\
\pm 0.67\end{array}$ & $\begin{array}{c}800.50 \pm \\
245.72\end{array}$ & $\begin{array}{l}46.66 \\
\pm 1.55\end{array}$ & $\begin{array}{l}15.51 \\
\pm 0.42\end{array}$ & $\begin{array}{l}33.13 \\
\pm 0.69\end{array}$ & $\begin{array}{r}984.690 \\
\pm 241.74\end{array}$ & $\begin{array}{l}47.29 \\
\pm 1.48\end{array}$ & $\begin{array}{l}15.80 \\
\pm 0.50\end{array}$ & $\begin{array}{c}33.3 \pm \\
0.38\end{array}$ & $\begin{array}{c}826.49 \pm \\
250.3\end{array}$ & $\begin{array}{l}47.31 \\
\pm 1.68\end{array}$ & $\begin{array}{l}18.51 \\
\pm 8.86\end{array}$ & $\begin{array}{l}31.21 \\
\pm 0.59\end{array}$ & $\begin{array}{l}847.973 \\
\pm 168.16\end{array}$ \\
\hline $\begin{array}{c}30 \\
\text { days }\end{array}$ & $\begin{array}{l}46.97 \\
\pm 1.16\end{array}$ & $\begin{array}{l}15.52 \\
\pm 0.45\end{array}$ & $\begin{array}{l}33.04 \\
\pm 0.50\end{array}$ & $\begin{array}{c}757.50 \pm \\
176.64\end{array}$ & $\begin{array}{l}47.44 \\
\pm 2.32\end{array}$ & $\begin{array}{l}15.65 \\
\pm 0.99\end{array}$ & $\begin{array}{l}33.03 \\
\pm 0.61\end{array}$ & $\begin{array}{r}1066.80 \\
\pm 356.46\end{array}$ & $\begin{array}{l}48.86 \\
\pm 4.21\end{array}$ & $\begin{array}{l}16.07 \\
\pm 1.20\end{array}$ & $\begin{array}{l}33.13 \\
\pm 0.96\end{array}$ & $\begin{array}{r}1085.80 \\
\pm 151.69\end{array}$ & $\begin{array}{l}47.17 \\
\pm 1.37\end{array}$ & $\begin{array}{r}15.79 \\
\pm 0.62\end{array}$ & $\begin{array}{l}33.53 \\
\pm 0.60\end{array}$ & $\begin{array}{l}895.50 \pm \\
211.31\end{array}$ \\
\hline $\begin{array}{c}60 \\
\text { day }\end{array}$ & $\begin{array}{l}47.03 \\
\pm 0.82\end{array}$ & $\begin{array}{l}15.68 \\
\pm 0.40\end{array}$ & $\begin{array}{l}33.32 \\
\pm 1.23\end{array}$ & $\begin{array}{l}1155.60 \\
\pm 210.81\end{array}$ & $\begin{array}{l}47.54 \\
\pm 1.61\end{array}$ & $\begin{array}{l}16.12 \\
\pm 0.55\end{array}$ & $\begin{array}{l}33.95 \\
\pm 0.52\end{array}$ & $\begin{array}{r}1195.60 \\
\pm 252.85\end{array}$ & $\begin{array}{l}47.29 \\
\pm 1.07\end{array}$ & $\begin{array}{l}15.78 \\
\pm 0.50\end{array}$ & $\begin{array}{l}33.52 \\
\pm 0.31\end{array}$ & $\begin{array}{r}1157.40 \\
\pm 224.48\end{array}$ & $\begin{array}{l}46.80 \\
\pm 1.82\end{array}$ & $\begin{array}{l}15.95 \\
\pm 0.75\end{array}$ & $\begin{array}{l}34.05 \\
\pm 0.71\end{array}$ & $\begin{array}{c}1337.0 \pm \\
133.88\end{array}$ \\
\hline 90 & $\begin{array}{l}47.37 \\
\pm 1.44\end{array}$ & $\begin{array}{l}16.00 \\
\pm 0.60\end{array}$ & $\begin{array}{l}33.76 \\
\pm 0.75\end{array}$ & $\begin{array}{r}1073.94 \\
\pm 438.94\end{array}$ & $\begin{array}{l}46.22 \\
\pm 1.96\end{array}$ & $\begin{array}{l}15.75 \\
\pm 0.70\end{array}$ & $\begin{array}{l}34.08 \\
\pm 0.50\end{array}$ & $\begin{array}{r}1280.50 \\
\pm 248.08\end{array}$ & $\begin{array}{l}47.79 \\
\pm 1.32\end{array}$ & $\begin{array}{l}16.40 \\
\pm 0.60\end{array}$ & $\begin{array}{l}34.35 \\
\pm 0.58\end{array}$ & $\begin{array}{l}1128.50 \\
\pm 219.81\end{array}$ & $\begin{array}{l}47.08 \\
\pm 1.89\end{array}$ & $\begin{array}{l}15.83 \\
\pm 0.47\end{array}$ & $\begin{array}{l}33.70 \\
\pm 0.65\end{array}$ & $\begin{array}{l}1154.10 \\
\pm 271.02\end{array}$ \\
\hline
\end{tabular}


In present investigation, the Acute and sub-chronic effects of rhGMCSF in mice as well as rabbits were presented. It has been observed that there are no toxicological symptoms in both acute and sub-chronic toxicity at predefined dosage levels in all the treated groups in both mice and rabbits. Based on the acute toxicity study for 14 days, it was found that $\mathrm{LD}_{50}$ in mice was $325 \mu \mathrm{g} / 20 \mathrm{~g}$ mice.

Further, the sub chronic toxicity test in mice was conducted after the observation of non-lethality of single dose of $325 \mu \mathrm{g}$ rhGM-CSF/20 g mice in acute toxicity study. 30 day repeated dose toxicology study with recovery groups revealed the safety profile of rhGM-CSF. Taking this into consideration we studied the safety profile of the rhGM-CSF for longer duration of exposure, i.e., 90 day subchronic toxicity study. The animals exposed to the rhGMCSF during 90 day sub-chronic toxicity study did not show any abnormalities in behavior and physical activities. The gain in body weights, between exposed animals to rhGMCSF and respective control group animals was not significant (Figure 1).

In addition, qualitative tests in urine also did not shown any significant abnormalities. Further, blood glucose, protein levels, kidney and liver function tests including electrolytes (Calcium, Magnesium and Potassium) were found to be in normal range and no significant changes observed in various group of animals exposed to the rhGMCSF and vehicle control. The hematological parameters were also found to be within normal range immediately after exposure and post exposure to test compound (Tables 3 and 4) in both mice and rabbits. There were no histopathological changes in various organs viz. heart, lung, brain and kidney in the mice exposed to the rhGMCSF. Based on the all these results, it has been revealed that there are no abnormalities in behavior, clinical, hematological or immunopathological and histopathological observations at all recommended therapeutic dose and higher doses under the experimental conditions. Based on these results, the present investigation suggested rhGM-CSF is safe and further conducting the clinical studies might be helpful for using the rhGM-CSF as a therapeutic drug.

\section{Acknowledgment}

Authors are gratefully acknowledged the help; valuable suggestions and guidance rendered by the Food and Toxicology Research Centre and the Department of Pathology of National Institute of Nutrition, Hyderabad. We thank the scientific staff (Study Personnel) mentioned in the report for their active involvement in the project. All other administrative staff at Virchow Biotech Pvt Ltd that has provided direct or indirect assistance is also gratefully acknowledged. Finally a special mention is reserved for the CPCSEA and all those animals whose involuntary sacrifice has made the progress of this study a reality.

\section{References}

Andrew J. Hapel and Richard E. Stanley. Cytokines, Receptors and Signalling Pathways Involved in Macrophage and Dendritic Cell Development, Madame Curie Bioscience Database. Austin (TX): Landes Bioscience; 2000-2013.

Bajji Chitra, Ravikanth Reddy Kosana, Radha Madhavi Dandu, Murali Krishna Reddy Tummuru, Increased periplasmic expression and purification of biologically active recombinant human granulocytemacrophage colony-stimulating factor from Escherichia coli. 2017, (Manuscript under review)

Ballinger MN, Paine R, 3rd, CH Serezani, DM Aronoff, ES Choi, et al (2006) Role of granulocyte macrophage colony-stimulating factor during gramnegative lung infection with Pseudomonas aeruginosa. American journal of respiratory cell and molecular biology 34: 766-774.

Cashen AF, Link D, Devine S, et al. Cytokines and stem cell mobilization for autologous and allogeneic transplantation. Curr. Hematol Rep. 2004; 3: 406-12.

Fantuzzi G. Cytokine Knockouts. New Jersey: Humana Press; 2003.

Ferlazzo G, Klein J, Paliard X, et al. Dendritic cells generated from CD34+ progenitor cells with flt3 ligand, c-kit ligand, GM-CSF, IL4, and TNF-alpha are functional antigen-presenting cells resembling monocyte-derived dendritic cells. Journal of Immunotherapy. 2000;23:48-58.

Griffiths S A and Lumley C E, Safety Evaluation of Biotechnologically-derived Pharmaceuticals, Facilitating a Scientific Approach, Centre for Medicines Research International, Carshalton, Surrey, UK, 1997, Page No.71-72.

Guthridge M.A et al., Mechanism of activation of the GM-CSF, IL-3, and IL-5 family of receptors. Stem cells, 1998. 16 (5): p. 301-13.

Hepatitis C: global Prevalence. Weekly Epidemiological Record, 1997, 72 (46):341-344.

Indian Pediatrics, 2001; 38: 1318-1322

Kane M Country specific hepatitis B carrier rates, by world countries and regions. WHO/EPI, Geneva, 1998 (Unpublished)

Kaushansky K, Broudy V. C, Harlan J. M, and Adamson. 1988. Tumor necrosis factor- a and tumor J. W necrosis factor-b (lymphotoxin) stimulate the 
production of granulocyte-macrophage colonystimulating factor, macrophage colony-stimulating factor, and IL-1 in vivo. J. Immunol. 141: 3410-3415

Lanza F, Rigolin G M, Castagnari B, Moretti S, Castoldi G, Potential clinical applications of rhGM-CSF in Acute Myeloid Leukemia Based on Its Biologic Activity and Receptor Interaction Haematologica 82 (2), 239-245. Mar-Apr 1997.

Metcalf D. Cell - cell signaling in the regulation of blood cell formation and function. Immunol Cell Biol. 1998; 76:441-7.

Paine R, $3^{\text {rd }}$, Preston AM, Wilcoxen S, Jin H, Siu BB, et al (2000) Granulocyte-macrophage colony-stimulating factor in the innate immune response to Pneumocystis carinii pneumonia in mice. J Immunol 164: 2602-2609.

Schulz Gregor M.D, Frisch Jurgen Greifenberg M.D, Berthold M.D. Nicolay Uwe, M.S, Oster Wolfgang M.D. New Therapeutic Modalities for the Clinical Use of rhGM-CSF in Patients with Malignancies. American Journal of Clinical Oncology: 1991
Sonoda Y et al., Erythroid burst-promoting activity of purified recombinant human GM-CSF and interleukin-3: studies with anti-GM-CSF and anti-IL3 sera and studies in serum-free cultures. Blood, 1988. 72(4): p. 1381-6.

Sechdule Y, Drugs and Cosmetics Rules, 1945

The HIV/AIDS Pandemic: 1993 Overview. Geneva, World Health Organization, 1993 (Unpublished document WHO/GPA/CNP/EVA/93.1).

Veronique Gautier. Jean-Louis Pujol, Francois-Bernard Michel, Interleukin-1 $\alpha$ and soluble interleukin-2 receptor during small cell lung cancer chemotherapy: comparison of high chemotherapy dose with rhGMCSF and standard chemotherapy dose without rhGMCSF, Lung Cancer, Volume 13, Issue 2, October 1995, Pages 145-153.

Zhang A.L et al., Natural killer cells trigger differentiation of monocytes into dendritic cells. Blood, 2007, 110 (7): p. 2484-93.

\section{How to cite this article:}

Bajji Chitra, Durga Bhavani Konga, Pavan Kumar Manikonda, P. Sudhakar, K.R.S. Sambha Siva Rao, Murali Krishna Reddy Tummuru. 2017. Pre-clinical Safety Evaluation of Recombinant Human Granulocyte Macrophage Colony Stimulating Factor (rh GM-CSF). Int.J.Curr.Res.Aca.Rev. 5(6), 28-37.

doi: https://doi.org/10.20546/ijcrar.2017.506.004 